\title{
Adiabatic creation of coherent superposition states via multiple intermediate states
}

\author{
A. Karpati and Z. Kis \\ Department of Nonlinear and Quantum Optics, \\ Research Institute for Solid State Physics and Optics, \\ Hungarian Academy of Sciences, \\ P.O. Box 49, H-1525 Budapest, Hungary
}

(Dated: November 10, 2018)

\begin{abstract}
We consider an adiabatic population transfer process that resembles the well established stimulated Raman adiabatic passage (STIRAP). In our system, the states have nonzero angular momentums $J$, therefore, the coupling laser fields induce transitions among the magnetic sublevels of the states. In particular, we discuss the possibility of creating coherent superposition states in a system with coupling pattern $J=0 \Leftrightarrow J=1$ and $J=1 \Leftrightarrow J=2$. Initially, the system is in the $J=0$ state. We show that by two delayed, overlapping laser pulses it is possible to create any final superposition state of the magnetic sublevels $|2,-2\rangle,|2,0\rangle,|2,+2\rangle$. Moreover, we find that the relative phases of the applied pulses influence not only the phases of the final superposition state but the probability amplitudes as well. We show that if we fix the shape and the time-delay between the pulses, the final state space can be entirely covered by varying the polarizations and relative phases of the two pulses. Performing numerical simulations we find that our transfer process is nearly adiabatic for the whole parameter set.
\end{abstract}

PACS numbers: 42.50.Hz, 42.65.Dr, 32.80.Bx

\section{INTRODUCTION}

Adiabatic methods in quantum mechanics play a fundamental role in the treatment of level crossing problems. The famous Landau-Zener system [1] has been followed by several other models which were defined in two-level systems [2, 3, 4, [5]. These models induced many applications in atomic and molecular physics, for recent review see Ref. 6].

It is common in all of the adiabatic systems that the Hamiltonian is time-dependent. The instantaneous eigenstates define an adiabatic basis. Suppose that at the initial time, one of the adiabatic states coincides with the initial state of the system. In most cases the adiabatic state that belongs to the eigenvalue zero is utilized for the transfer. If the system varies with time slowly enough, then its state vector follows adiabatically the adiabatic eigenstate and it will go over smoothly to the desired final state. The adiabaticity of the process guarantees its robustness with respect to fluctuations in the parameters of the couplings. In the limit of ideal adiabatic transfer, the other eigenstates are not invoked in the evolution and their population remains zero throughout the whole time. The non-adiabatic corrections tend to involve these states, but the magnitudes of such couplings are supposed to be small compared with their energy separation from the adiabatic states.

During the last decade, a promising new population transfer technique was introduced: the stimulated Raman adiabatic passage (STIRAP) process realizes efficient population transfer in three-level systems, see Ref. [7] and references therein. Moreover, it turned out that this scheme can be applied successfully to the problem of manipulating and creating coherent state superpositions.
Such superpositions are the desired initial states for many modern quantum applications including information processing and communication. The original STIRAP process has thus been utilized to create coherent superpositions in three- and four-level systems $8,9,10,11,12$ and to prepare $N$-component maximally coherent superposition states 13]. The four-level (tripod) STIRAP establishes such applications as qubit rotation 14 and densitymatrix measurement [15].

Recently, a multilevel STIRAP scheme has been proposed 16, 17 to create $N$-level coherent superposition states. This model is particularly interesting, since the Hamiltonian has several adiabatic eigenstates which belong to the eigenvalue zero. The diabatic couplings among these eigenstates influence significantly the dynamics of the system. However, the eigenstates in the zero eigenvalue subspace are decoupled from the eigenstates belonging to nonzero eigenvalues provided the time-evolution is adiabatic. In Ref. [16] the Optimal Control Theory has been applied to find the optimal pulse sequences which create a prescribed final superposition state. It has also been verified that the transfer process occurs in the zero-eigenvalue subspace. In a subsequent work 17] an analytic solution has been presented for a five-level system.

When the STIRAP concept is realized in atoms or molecules one encounters that the quantum states with angular momentum $J$ have magnetic sublevels and these sublevels are coupled by the applied laser pulses, and a rather complicated coupling-pattern may occur. This situation has been studied in Refs. 18, 19, 20]. In these papers the authors have discussed the possibility of transferring population from a well defined initial state to a single but arbitrarily chosen magnetic sublevel.

An other realistic problem is the presence of multiple 
or continuum intermediate states $21,22,23,24,25,26$, 27, 28]. In Ref. 21] the continuum has been modeled by infinite number of equidistant discrete levels which were coupled by unique couplings to a single initial and final state. Later, it has been shown that the previous model is too simple, the physics is more complicated in a realistic situation [22]. A detailed study of the discrete model has been presented in Ref. 23. It has also been shown that efficient population transfer is indeed possible through a continuum 24, 25, 26, 27, 28].

In this paper we will study a multilevel $\Lambda$ scheme similar to the one discussed in Refs. 18, 19, 20]. However, in contrast with the previous works where the population transfer took place between a single initial and a single final state, our aim is to create a multilevel final superposition state. In our model, the initial state has angular momentum $J=0$, the intermediate state has $J=1$ and the final superposition state is formed from the magnetic sublevels of a state $J=2$. We will design a STIRAP-like process, i.e. the population transfer is realized by delayed, overlapping laser pulses. We define the Hamiltonian corresponding to the process, separate the adiabatic states belonging to the eigenvalue zero and find the conditions for the successful population transfer. We will study how does the final state depend on the relative phases of the applied laser pulses. The robustness of the transfer process will also be discussed.

The organization of the paper is as follows: In Section III the physical model of our six-level STIRAP is presented and the corresponding Hamiltonian is introduced. The Hilbert space is separated to a zero-eigenvalue subspace and to a nonzero-eigenvalue subspace. The eigenstates belonging to the zero eigenvalue are determined explicitly. In Section III we study the possibility of population transfer by means of delayed laser pulses. We show numerically that nearly adiabatic evolution is possible. We also discuss the robustness of the transfer process. We summarize the results in Section IV

\section{SIX-LEVEL SYSTEM}

Let us consider the six-level $\Lambda$ configuration shown in Fig. 11 The system consists of the magnetic sublevels of states with total angular momentum $J=0, J=1$ and $J=2$. The $J=1$ states have higher energy than the $J=0$ and $J=2$ states. Initially, only the $J=0$ state is populated. Our goal is to transfer this population to the sublevels of the $J=2$ state and to obtain a prescribed final superposition state. We are going to design a STIRAPlike population transfer process. To this end, we apply two sets of laser pulses, the corresponding Rabi frequencies are denoted by $\Omega_{S \pm}$ and $\Omega_{p \pm}$. The subscripts $S$ and $p$ refer to "Stokes" and "pulse", while the indices +/correspond to right- and left-hand circular polarizations, respectively. Obviously, in this configuration only the sublevels with $m=-2,0,+2$ can be populated in the final state. This linkage can be realized experimentally in Neon atoms, see Ref. [20]. The more general case, where all magnetic sublevels of the $J=2$ state are included into the final superposition state will be discussed elsewhere. The frequency and the phase of the laser pulses are kept constant during the interaction, only their amplitudes are time-dependent.

In our model we have to determine the Rabi frequencies in an angular-momentum basis 29]. According to the Wigner-Eckart theorem, the general formula for the Rabi frequency between two levels of angular momenta $J_{1}$ and $J_{2}$ and magnetic quantum numbers $M_{1}$ and $M_{2}$ is given by

$$
\begin{aligned}
\hbar \Omega\left(J_{1} M_{1} ; J_{2} M_{2}\right) & =-\mathcal{E}^{*}(-1)^{J_{1}-M_{1}}\left(J_{1}\|\hat{d}\| J_{2}\right) \\
& \times \sum_{s=-1}^{+1}(-1)^{s} \epsilon_{-s}\left[\begin{array}{ccc}
J_{1} & 1 & J_{2} \\
-M_{1} & s & M_{2}
\end{array}\right]
\end{aligned}
$$

where the electric field strength is $\mathcal{E}$, the reduced dipole matrix element is given by $\left(J_{1}\|\hat{d}\| J_{2}\right), \epsilon_{s}$ are the irreducible tensor operator components of the polarization vector of the field, and [...] denotes the $3-j$ symbol. We apply left- and right-hand circularly polarized light, therefore, in the summation $s= \pm 1$ and $\epsilon_{ \pm 1}=1$.

The two pump pulses couple the level $|00\rangle$ to the levels $|1, \pm 1\rangle$. The corresponding Rabi frequencies can be expressed as $\Omega_{p \pm}=$ const $_{1} \times \mathcal{E}_{p \pm}^{*}$. The right-hand circularly polarized Stokes pulse couple the levels $|1,-1\rangle \Leftrightarrow|2,-2\rangle$ and $|1,+1\rangle \Leftrightarrow|2,0\rangle$. For the Rabi frequencies we obtain

$$
\begin{aligned}
\Omega_{S+}(1,-1 ; 2,-2) & =q \times \text { const }_{2} \times \mathcal{E}_{S+}^{*}, \\
\Omega_{S+}(1,+1 ; 2,0) & =\text { const }_{2} \times \mathcal{E}_{S+}^{*},
\end{aligned}
$$

where $q$ is the ratio of the corresponding $3-j$ symbols. In our case $q=\sqrt{6}$. Similarly, the left-hand circularly polarized Stokes pulse couple the levels $|1,-1\rangle \Leftrightarrow|2,0\rangle$ and $|1,+1\rangle \Leftrightarrow|2,+2\rangle$, and the Rabi frequencies are given by

$$
\begin{aligned}
\Omega_{S-}(1,-1 ; 2,0) & =\text { const }_{2} \times \mathcal{E}_{S-}^{*} \\
\Omega_{S-}(1,+1 ; 2,+2) & =q \times \text { const }_{2} \times \mathcal{E}_{S-}^{*} .
\end{aligned}
$$

In summary, the Rabi frequencies can be expressed as

$$
\begin{aligned}
\Omega_{p+}(0,0 ; 1,-1) & =A \exp i \varphi_{A}, \\
\Omega_{p-}(0,0 ; 1,+1) & =B \exp i \varphi_{B}, \\
\Omega_{S+}(1,-1 ; 2,-2) & =q C \exp i \varphi_{C}, \\
\Omega_{S+}(1,+1 ; 2,0) & =C \exp i \varphi_{C}, \\
\Omega_{S-}(1,-1 ; 2,0) & =D \exp i \varphi_{D}, \\
\Omega_{S-}(1,+1 ; 2,+2) & =q D \exp i \varphi_{D} .
\end{aligned}
$$

The phases $\varphi_{X}$ are constants, and the envelope functions $X$ are non-negative. The laser pulses may be detuned from resonance by a frequency $\Delta$, but we require that they are at multi-photon resonance. The interaction Hamiltonian in the basis $\{|0,0\rangle,|1,-1\rangle,|1,+1\rangle,|2,-2\rangle$, $|2,0\rangle,|2,+2\rangle\}$ reads 


$$
H=\frac{1}{2} \hbar\left[\begin{array}{cccccc}
0 & A \exp i \varphi_{A} & B \exp i \varphi_{B} & 0 & 0 & 0 \\
A \exp -i \varphi_{A} & \Delta & 0 & q C \exp i \varphi_{C} & D \exp i \varphi_{D} & 0 \\
B \exp -i \varphi_{B} & 0 & \Delta & 0 & C \exp i \varphi_{C} & q D \exp i \varphi_{D} \\
0 & q C \exp -i \varphi_{C} & 0 & 0 & 0 & 0 \\
0 & D \exp -i \varphi_{D} & C \exp -i \varphi_{C} & 0 & 0 & 0 \\
0 & 0 & q D \exp -i \varphi_{D} & 0 & 0 & 0
\end{array}\right]
$$

The time-evolution of the system is governed by the Schrödinger equation

$$
i \hbar \frac{\partial}{\partial t}|\psi\rangle=H|\psi\rangle .
$$

In a STIRAP process, the Hilbert space is split to dark and bright subspaces and the system evolves in the dark subspace. These subspaces are formed of the instantaneous eigenstates of the time-dependent Hamiltonian. The dark states belong to the eigenvalue zero and they do not have projection to the excited state(s) [30]. The bright states belong to nonzero eigenvalues and they overlap with the excited state(s). In the adiabatic limit, the system evolves entirely in the dark subspace. Now we continue the analysis of the Hamiltonian Eq. (5) in this direction. First, we determine the dark and bright subspaces of the Hilbert space. By applying a diagonal unitary transformation $U_{1}$ to the system, the number of independent phases in the Hamiltonian can be reduced to one:

$$
H_{1}=U_{1} H U_{1}^{\dagger}=\frac{1}{2} \hbar\left[\begin{array}{cccccc}
0 & A & B e^{i \varphi} & 0 & 0 & 0 \\
A & \Delta & 0 & q C & D & 0 \\
B e^{-i \varphi} & 0 & \Delta & 0 & C & q D \\
0 & q C & 0 & 0 & 0 & 0 \\
0 & D & C & 0 & 0 & 0 \\
0 & 0 & q D & 0 & 0 & 0
\end{array}\right]
$$

where

$$
\begin{gathered}
U_{1}=\operatorname{diag}\left[e^{-i\left(\varphi_{A}+\varphi_{C}\right)}, e^{-i \varphi_{C}}, e^{i\left(\varphi_{D}-2 \varphi_{C}\right)}, 1,\right. \\
\left.e^{i\left(\varphi_{D}-\varphi_{C}\right)}, e^{i\left(2 \varphi_{D}-2 \varphi_{C}\right)}\right],
\end{gathered}
$$

and the remaining phase factor

$$
\varphi=\varphi_{B}-\varphi_{A}+\varphi_{C}-\varphi_{D} .
$$

It will turn out that this phase factor is indeed relevant, since it affects the probability amplitudes of the final state.

As a next step we are going to find a time-dependent unitary transformation that defines a new basis, in which the dark and bright subspaces of the Hilbert space are separated. By applying rotations and diagonal unitary transformations in a well-chosen order we arrive at the following unitary transformation:

$$
U=U_{4} U_{3} U_{2}
$$

where

$$
U_{2}=\left[\begin{array}{cccccc}
1 & 0 & 0 & 0 & 0 & 0 \\
0 & 1 & 0 & 0 & 0 & 0 \\
0 & 0 & 1 & 0 & 0 & 0 \\
0 & 0 & 0 & -\frac{y}{q x} & \frac{y k}{x} & -\frac{y k^{2}}{q x} \\
0 & 0 & 0 & \frac{y^{2} k}{q x} & \frac{1}{x} & \frac{y^{2} k^{3}}{q x} \\
0 & 0 & 0 & \frac{y k^{2}}{q} & 0 & -\frac{y}{q}
\end{array}\right]
$$

and

$$
\begin{gathered}
U_{3}=\operatorname{diag}[\exp i \xi, 1,1,1, \exp i \zeta, 1] \\
U_{4}=\left[\begin{array}{cccccc}
\cos \alpha & 0 & 0 & 0 & 0 & \sin \alpha \\
0 & 1 & 0 & 0 & 0 & 0 \\
0 & 0 & 1 & 0 & 0 & 0 \\
0 & 0 & 0 & 1 & 0 & 0 \\
-\sin \beta \sin \alpha & 0 & 0 & 0 & \cos \beta & \sin \beta \cos \alpha \\
-\cos \beta \sin \alpha & 0 & 0 & 0 & -\sin \beta & \cos \beta \cos \alpha
\end{array}\right]
\end{gathered}
$$

where we made use of the definitions

$$
\begin{aligned}
k & =C / D, \\
x & =\sqrt{k^{4}+k^{2} q^{2}+1} / \sqrt{k^{4}+1}, \\
y & =-q / \sqrt{k^{4}+1}, \\
\tan \alpha & =\frac{\left(k^{4}+1\right) y D}{\sqrt{A^{2} k^{2}+B^{2}-2 A B k \cos \varphi}}, \\
\tan \beta & =-\frac{y}{x} \cos \alpha \sqrt{\frac{A^{2}+B^{2} k^{6}+2 A B k^{3} \cos \varphi}{A^{2} k^{2}+B^{2}-2 A B k \cos \varphi}}(14 \mathrm{e}) \\
\xi & =\arctan \left(\frac{B \sin \varphi}{A k-B \cos \varphi}\right), \\
\zeta & =\xi+\arctan \left(\frac{B k^{3} \sin \varphi}{A+B k^{3} \cos \varphi}\right) .
\end{aligned}
$$

If we transform the Hamiltonian Eq. (7) with the unitary transformation $U$ in Eq. (10) we obtain

$$
\tilde{H}_{1}=U H_{1} U^{\dagger},
$$

which has two rows and two columns with zero elements. This means that the unitary transformation $U$ defines 
two dark states. The first one is given by the fourth row of $U_{2}$

$$
\left|D_{1}\right\rangle=\frac{1}{\sqrt{C^{4}+D^{4}+q^{2} C^{2} D^{2}}}\left[\begin{array}{c}
0 \\
0 \\
0 \\
D^{2} \\
-q C D \\
C^{2}
\end{array}\right] .
$$

The one-dimensional subspace corresponding to that dark state is not altered by the other unitary transformations $U_{3}$ and $U_{4}$, and it is a superposition of the levels with $J=2$. The sixth row of the matrix $U$ defines the second dark state

$$
\left|D_{2}\right\rangle=\left[\begin{array}{c}
-\cos \beta \sin \alpha \exp i \xi \\
0 \\
0 \\
-\frac{y^{2} k}{q x} \sin \beta \exp i \zeta+\frac{y k^{2}}{q} \cos \beta \cos \alpha \\
-\frac{1}{x} \sin \beta \exp i \zeta \\
-\frac{y^{2} k^{3}}{q x} \sin \beta \exp i \zeta-\frac{y}{q} \cos \beta \cos \alpha
\end{array}\right] .
$$

The states $\left|D_{1}\right\rangle$ and $\left|D_{2}\right\rangle$ are indeed dark states, since they do not have components among the $J=1$ levels.

To complete the study of the dark and bright subspaces of our system we should diagonalize completely the Hamiltonian $\tilde{H}_{1}$ in Eq. (15). This leads to rather involved formulae, however, for our purposes it is enough to show that the eigenvalues of the $4 \times 4$ nonzero block of $\tilde{H}_{1}$ are nonzero and the corresponding eigenstates are bright states. We have verified numerically using Maple [31] that the previous assumptions are true. We conclude that the states given by Eqs. (16) and (17) are the two dark states of our system and it has four bright states. The dark subspace is degenerate, so particular attention should be payed when one studies the time-evolution of the system [9, 16, 17]. In general, for time-dependent dark states there appear a nonadiabatic coupling term in the transformed Hamiltonian which couple the dark states. We will return to this issue in the Sec. III We can transform the Schrödinger equation (6) to the timedependent basis defined by $U_{1}$ and $U$ in Eqs. (8) and (10)

$$
i \hbar \frac{\partial}{\partial t}|\tilde{\psi}\rangle=\tilde{H}|\tilde{\psi}\rangle,
$$

through the relations

$$
\begin{aligned}
|\tilde{\psi}\rangle & =U U_{1}|\psi\rangle, \\
\tilde{H} & =\tilde{H}_{1}+i \dot{U} U^{\dagger},
\end{aligned}
$$

where $\tilde{H}_{1}$ is given by Eq. (15). In this form the dark and bright subspaces are separated. Since we want to design a STIRAP-like population transfer process, in the following we focus our attention to the dark states. The bright states are needed when one wants to study the coupling between the dark and bright subspaces, but since the formulae for the bright states are too complicated, we shall do it in an other way.

\section{ADIABATIC POPULATION TRANSFER}

Now we make a further assumption: We assume that the ratios $A / B$ and $C / D$ are constant during the timeevolution, i.e. the envelope function of the two pump pulses are the same, only their amplitudes and phases are different. Similarly for the Stokes pulses. Consequently, the parameter $k$ defined in Eq. (14a) is constant. Therefore, the dark state $\left|D_{1}\right\rangle$ is constant throughout the whole time. This simplifies considerably the further analysis. It follows immediately, that though the dark subspace is degenerate, the two dark states do not mix with each-other during the interaction, since $\left\langle D_{2} \mid \dot{D}_{1}\right\rangle=0$. Therefore, the Hamiltonian $\tilde{H}$ has a $2 \times 2$ zero matrix block acting on the dark subspace.

The dark state $\left|D_{2}\right\rangle$ has components among the $J=0$ and $J=2$ states as well. Hence, it is a good candidate to realize the transfer process under consideration. Initially the system is in the state $|0,0\rangle$. For implementing a STIRAP process, that state should be a dark state of the system. Since the state $\left|D_{1}\right\rangle$ cannot participate in a superposition forming the initial state, we require $\left|D_{2}\right\rangle$ to coincide with the state $|0,0\rangle$ at early times. This condition is satisfied if $C^{2}+D^{2} \gg A^{2}+B^{2}$, which means that the pulses $C$ and $D$ arrive before $A$ and $B$. Though we have not presented explicitly the bright states of our system, it is sure that they have no projection to the initial state, since they are always orthogonal to the dark states and $\left|D_{2}\right\rangle \equiv|0,0\rangle$ initially, if the above condition is fulfilled. This argument ensures that the initial state of the system lays in the dark subspace which is necessary for a STIRAP process.

We have required that the final state of the system is a linear combination of the levels with $J=2$ and $m=-2,0,+2$. The dark state $\left|D_{2}\right\rangle$ has these components as well, so the previous condition is true only if the first element of the state $\left|D_{2}\right\rangle$ is zero at the end of the adiabatic evolution. Consequently, at late times it is eligible that $C^{2}+D^{2} \ll A^{2}+B^{2}$, therefore, the pulses $C$ and $D$ terminate before $A$ and $B$.

The previous conditions suggest that for successful population transfer we need counterintuitive time ordering of the pump and Stokes pulses, similarly to the original STIRAP. Now we consider the possibility of adiabatic population transfer using the connectivity argument of Ref. [19]: The system Hamiltonian has a constant zero eigenvalue which is degenerate. However, as we have shown in the beginning of this Section, the nonadiabatic coupling between the two degenerate dark states vanishes because one of them is constant. As we have seen in the previous paragraphs, the time dependent dark state coincides with the initial state of the system at the beginning of the process when only the Stokes pulse is present. Similarly, this dark state lays in the required final state subspace at the end of the process when only the pump pulse is present. Therefore, the connectivity requirements are satisfied 19], we have got a dark state that connects smoothly the initial state of the system 
with the final state. However, besides these conditions we have to fulfill adiabaticity as well. We will discuss this issue below.

The considered six-level system can be regarded as four coupled three-level systems: For $A=0$ and $C=0$ the pulses $B$ and $D$ define a three-level STIRAP process between the levels $|0,0\rangle,|1,+1\rangle$ and $|2,0\rangle$. For $A=0$ and $D=0$ the pulses $B$ and $C$ define a three-level STIRAP process between the levels $|0,0\rangle,|1,+1\rangle$ and $|2,+2\rangle$. For $B=0, C=0$ and $B=0, D=0$ the situation is similar. The envelopes $A, B, C, D$ can be parametrized in a form that emphasizes the presented qualitative picture:

$$
\begin{aligned}
A & =R_{p} \cos \eta \\
B & =R_{p} \sin \eta \\
C & =\frac{R_{S}}{\sqrt{1+q^{2}}} \cos \nu \\
D & =\frac{R_{S}}{\sqrt{1+q^{2}}} \sin \nu
\end{aligned}
$$

where the constant angles $\eta$ and $\nu$ are in the interval $[0, \pi / 2]$. These angles define the polarization of the laser pulses. The six-level system simplifies to a three-level $\Lambda$ system when $\eta, \nu=0, \pi / 2$. For arbitrary constant angles $\eta$ and $\nu$ fixed couplings exist between the individual three-level STIRAP processes.

If the time-evolution is adiabatic, then the dark and bright subspaces do not mix during the whole interaction time [9, 16, 17]. Therefore, if the initial state of the system lays in the dark subspace, it will remain there. The physical consequence of the adiabatic evolution is that the excited states $|1, \pm 1\rangle$ are only minimally populated throughout the transfer process. As we saw in the previous paragraph, when $\eta, \nu=0, \pi / 2$ the system reduces to the ordinary three-level STIRAP. In this case the adiabaticity constraints are simple: Let us take $\eta, \nu=0, \pi / 2$, then adiabaticity requires

$$
\left|\dot{\vartheta} \frac{\sin ^{2} \varrho}{\cos \varrho}\right| \ll \frac{1}{2} \Omega, \quad\left|\dot{\vartheta} \frac{\cos ^{2} \varrho}{\sin \varrho}\right| \ll \frac{1}{2} \Omega,
$$

where $\Omega=\sqrt{\Omega_{p}^{2}+\kappa^{2} \Omega_{S}^{2}}, \tan \vartheta=\Omega_{p} / \kappa \Omega_{S},(\kappa=1, q)$, and $\tan 2 \varrho=\Omega / \Delta$. These conditions say that we need large pulse amplitudes and smooth, long pulses. For a complete study we would need the explicit form of the bright states $\left|B_{k}\right\rangle, k=1 \ldots 4$ of the Hamiltonian in Eq. (7). Then, adiabaticity requires to satisfy the following four equations:

$$
\left|\left\langle D_{2} \mid \dot{B}_{k}\right\rangle\right| \ll\left|\varepsilon_{k}-\varepsilon_{0}\right|, \quad k=1 \ldots 4,
$$

where $\varepsilon_{k}$ is the adiabatic eigenenergy associated with $\left|B_{k}\right\rangle$, and $\varepsilon_{0}=0$ belongs to the dark state $\left|D_{2}\right\rangle$. These equations yield the necessary and sufficient conditions for the pulses to be fulfilled in order that the evolution satisfy adiabaticity. Although the bright states $\left|B_{k}\right\rangle$ are not available analytically, these equations can be used to test adiabaticity numerically for a certain choice of the pulses. In the numerical simulations presented below we used these equations to test adiabaticity. Besides these conditions, below we show that a simplified approach is also possible: we formulate a sufficient condition for adiabatic evolution in the six-level system.

Our physical intuition suggests that if the three-level STIRAP processes for the special polarizations $\eta, \nu=$ $0, \pi / 2$ are adiabatic, then the six-level STIRAP process will also be adiabatic for constant angles $\eta$ and $\nu$. This is a sufficient condition for adiabaticity. It is independent of the polarization of the pulses, in fact, we apply the adiabaticity condition of the original three-level STIRAP to our six-level scheme. We have performed numerical simulations to reveal whether this assumption is valid or not.

In Figs. 2 and 3 the numerically calculated time evolution of the populations are shown for two different parameter sets. The pulses are Gaussian. The two parameter sets differ only in the phase $\varphi$. The final populations, denoted by $f_{1}, f_{2}$ and $f_{3}$ in the figures, are different, hence the phase $\varphi$ has indeed impact on the magnitude of the probability amplitudes in the final state. We interpret this phase dependence as a manifestation of quantum interference among the rivaling coupled population transfer processes.

To test adiabaticity in the previous examples, we evaluated Eqs. (22) for the parameters of Fig. 2] The results are shown in Fig. 4. It can be seen that for that timeinterval when adiabaticity is expected the conditions are well fulfilled. The non-adiabaticity of our six-level STIRAP process can be also quantified by the maximum population of the bright subspace during time-evolution. This non-adiabaticity is 0.014 and 0.011 for the two presented processes. The small values show that the mixing between the dark and bright subspaces is negligible, therefore, the time-evolution of the system is adiabatic. The efficiency of the population transfer can be defined as the sum of the norm of the final states with $J=2$. For the two presented processes it is found to be $99.98 \%$ and $99.97 \%$, showing that it is possible to perform a STIRAP process in the considered six-level system.

The final state of the system in the adiabatic limit can be obtained analytically from the expressions of the unitary transformations $U$ and $U_{1}$ at the beginning and at the end of the population transfer

$$
\left|\psi_{f}\right\rangle=U_{1}^{\dagger} U_{f}^{\dagger} U_{i} U_{1}\left|\psi_{i}\right\rangle
$$

provided that $\left|\psi_{i}\right\rangle$ lays in the dark subspace. In our case the initial state of the system is $\left|\psi_{i}\right\rangle=|0,0\rangle$. After some 
calculation we obtain

$$
\left|\psi_{f}\right\rangle=\left[\begin{array}{c}
0 \\
0 \\
0 \\
\left(-\frac{y^{2} k}{q x} \sin \beta e^{-i \zeta}+\frac{y k^{2}}{q} \cos \beta\right) e^{-i\left(\varphi_{A}+\varphi_{C}-\xi\right)} \\
\frac{1}{x} \sin \beta e^{-i\left(\varphi_{A}+\varphi_{D}-\xi\right)} \\
\left(-\frac{y^{2} k^{3}}{q x} \sin \beta e^{-i \zeta}-\frac{y}{q} \cos \beta\right) e^{-i\left(\varphi_{A}-\varphi_{C}+2 \varphi_{D}-\xi\right)}
\end{array}\right],
$$

where the parameters $k, x, y, \beta, \zeta, \xi$ are defined by Eqs. (14), and the parameters $\beta, \zeta$, and $\xi$ are evaluated at the final time. The equations (23) and (24) together with (14) show that our population transfer process is robust with respect to small fluctuations of the experimental parameters such as pulse shapes and pulse areas. However, the relative phases of the pulses influence not only the phases in the final superposition state but the probability amplitudes as well.

It is necessary to verify that all possible linear combinations of the states with $J=2$ and $m=-2,0,+2$ can be created by the considered STIRAP process. Suppose, we have a prescribed final state

$$
\left|\psi_{c}\right\rangle=\left[\begin{array}{c}
0 \\
0 \\
0 \\
c_{1} e^{i \varphi_{1}} \\
c_{2} e^{i \varphi_{2}} \\
c_{3} e^{i \varphi_{3}}
\end{array}\right]
$$

and we have found a pulse set with $\varphi_{A}=0, \varphi_{B}=\varphi$, $\varphi_{C}=0, \varphi_{D}=0$ for which the final populations of the state $\left|\psi_{f}\right\rangle$ are the same as in the prescribed state, and the equation

$$
\begin{aligned}
\varphi_{1}+\varphi_{3}-2 \varphi_{2} \equiv & \arg \left|\psi_{f}\right\rangle_{4}+\arg \left|\psi_{f}\right\rangle_{6}- \\
& -2 \arg \left|\psi_{f}\right\rangle_{5} \bmod 2 \pi
\end{aligned}
$$

holds. By adjusting the phases of the pulses to

$$
\begin{aligned}
\varphi_{A} & =\arg \left|\psi_{f}\right\rangle_{5}-\varphi_{2}, \\
\varphi_{B} & =\varphi+\arg \left|\psi_{f}\right\rangle_{6}-\varphi_{3}, \\
\varphi_{C} & =\arg \left|\psi_{f}\right\rangle_{5}-\arg \left|\psi_{f}\right\rangle_{6}+\varphi_{3}-\varphi_{2} \\
\varphi_{D} & =0
\end{aligned}
$$

the phases of the final state change to the phases of the prescribed state. The phase $\varphi$ defined by Eq. (9) is invariant under the presented phase adjustment, so the final populations remain the same. It follows that for deciding whether all prescribed final states can be reached, it is enough to show that all possible amplitudes $\left(c_{1}, c_{2}, c_{3}\right)$ and all possible values of

$$
\delta=\varphi_{1}+\varphi_{3}-2 \varphi_{2} \quad \bmod 2 \pi
$$

can be reached by pulse sets with $\varphi_{B}=\varphi$ and $\varphi_{A}=$ $\varphi_{C}=\varphi_{D}=0$.

The amplitudes $\left(c_{1}, c_{2}, c_{3}\right)$ satisfy $c_{1}^{2}+c_{2}^{2}+c_{3}^{2}=1$. This triplet defines a point on the one-eights of the surface of the unit sphere, since the restriction $c_{1}, c_{2}, c_{3} \geq 0$ must be satisfied. The surface points can be parametrized by two polar angles $\theta \in[0, \pi / 2]$ and $\chi \in[0, \pi / 2]$, therefore we have

$$
\begin{aligned}
& c_{1}=\cos \theta, \\
& c_{2}=\sin \theta \cos \chi, \\
& c_{3}=\sin \theta \sin \chi .
\end{aligned}
$$

If we fix the shape of the pulses and the time-delay between them, then we have three independent parameters which can be adjusted freely: the polarizations $\eta$ and $\nu$ and the combination $\varphi$ of their relative phases. The final state can be characterized by the triplet $(\theta, \chi, \delta)$. Mathematically, our population transfer process assigns to each triplet $(\eta, \nu, \varphi)$ a triplet $(\theta, \chi, \delta)$. If these points fill the cube $[0 \ldots \pi / 2,0 \ldots \pi / 2,0 \ldots 2 \pi]$, then all prescribed states can be reached by choosing a corresponding triplet $(\eta, \nu, \varphi)$ and performing the transformation defined in Eq. (27) on the initial phases of the pulses.

We have performed numerical simulations in order to answer the above question. The angles $\eta, \nu$ and $\varphi$ were incremented by 0.022 in their domain range. We have used the envelope functions $R_{p}=15 \exp [-((t-$ 1.8)/2.82 $\left.)^{2}\right]$ and $R_{S}=15 \sqrt{1+q^{2}} \exp \left[-((t+1.8) / 2.82)^{2}\right]$ for the pump and Stokes pulses, respectively. We have verified that these pulses fulfill the adiabaticity conditions Eq. (21) for the field polarizations $\eta, \nu=0, \pi / 2$. The resulting triplet set was divided into slices of width 0.1 in $\delta$. Each slice was checked whether it is filled with points. Some of the slices are shown in Figs. [5 6] 78 9. 10. We have found that each slice is equally and quite uniformly filled with points.

We have compared the numerical results with the analytical solution given by Eq. (23). Note that the analytical solution is valid in the adiabatic limit. In this case the increments of the parameters $\eta, \nu$ and $\varphi$ were 0.015 , giving a more detailed picture about the distribution of the resulting points $(\theta, \chi, \delta)$. The differences between the numerical simulation and analytical solution were small, below one percent, showing that the chosen pulse-sets realized a nearly adiabatic evolution. In Fig. 8 we show a slice of the distribution for the same parameter set as in Fig. 7

The maximum non-adiabaticity, that we measure as the maximum population of the bright subspace, encountered during the simulation was 0.006 . We have also verified that the adiabaticity conditions Eq. (22) are fulfilled for the whole parameter range. Therefore, our assumption is indeed true: if the population transfer process is adiabatic for some special polarizations of the pulses $\eta, \nu=0, \pi / 2$, i.e. we have an effective three-level system, then it remains adiabatic for arbitrary polarizations, i.e. all the six states are coupled. This is a sufficient condition for the pulses to satisfy adiabaticity. Based on the results of the numerical simulation and the analytical solution, we conclude that all prescribed states can be reached by the presented STIRAP-like population transfer process and the evolution is adiabatic. 


\section{SUMMARY}

In this paper we have discussed a STIRAP-like population transfer process in a six-level $\Lambda$ system. The initial state has angular momentum $J=0$, the excited state has $J=1$ and the final state has $J=2$. Our aim is to create coherent superposition states on the magnetic sublevels of the final state. Similarly to the original STIRAP, the couplings are realized by a pump and a Stokes laser pulse, which are elliptically polarized in our case. The shape of the pulses and the time-delay between them are fixed. However, their polarizations and the relative phases are freely adjustable but also fixed throughout the whole time. We have defined the Hamiltonian of this system in the RWA approximation. We have found that by time-independent unitary transformations not all the phases can be eliminated from the Hamiltonian, there remains one. This phase factor proved to be indeed relevant, since it influences the probability amplitudes in the final superposition state. We have determined the dark and bright subspaces of our system and we have given explicitly the two dark states. We have shown that the desired population transfer can be realized by a counterintuitive pulse sequence similarly to the original STIRAP. We have studied the conditions for adiabatic evolution in our system: In the case of special polarizations of the pulses the coupling pattern reduces to a simple three-level system. The adiabaticity conditions are well known for this case. We have shown numerically that the system evolves adiabatically for general polarizations as well if adiabaticity prevails for the special choice of the polarizations. We have compared the results of the simulation with the analytically calculated final states in the adiabatic limit, and an excellent agreement has been found. We have verified numerically that the whole final state space can be covered by varying the relative phases and the polarizations of the two exciting pulses, while the pulse shapes and the time-delay between them are fixed.

As we mentioned before, the probability amplitudes in the final state depend on the relative phases of the laser pulses. This is unusual in case of the STIRAP process, up to our knowledge that is the first time when the relative phases do matter. The phase dependence results from quantum interference, because the coupling pattern can be considered as a coupled set of three-level STIRAP systems. Therefore, the final state emerges from the rivaling STIRAP processes.

Finally, we comment briefly the experimental realization of our scheme: It seems easy to find an atomic system where the coupling pattern can be attained. Two elliptically polarized pulses are required, however, the phase shift between the left- and right-rotating components should be adjustable. Practically this seems the only additional experimental effort to be done compared with the realization of the traditional three-level STIRAP process.

\section{Acknowledgments}

This work was supported by the European Union Research and Training Network COCOMO, contract HPRN-CT-1999-00129. Z.K. acknowledges the support of the János Bolyai program of the Hungarian Academy of Sciences. A.K. acknowledges the support of the Research Fund of Hungary under contract No. T034484.
[1] L.D. Landau, Phys. Z. Sowjetunion 2, 46 (1932); C. Zener, Proc. R. Soc. London Ser. A 137, 696 (1932).

[2] N. Rosen and C. Zener, Phys. Rev. 40, 502 (1932).

[3] Yu.N. Demkov and M. Kunike, Vestn Leningr. Univ. Fis. Khim. 16, 39 (1969).

[4] F.T. Hioe, Phys. Rev. A 30, 2100 (1984).

[5] K.-A. Suominen, B.M. Garraway, and S. Stenholm, Opt. Commun. 82, 260 (1991).

[6] N.V. Vitanov, T. Halfmann, B.W. Shore, and K. Bergmann, Annu. Rev. Phys. Chem. 52, 763 (2001).

[7] N.V. Vitanov, M. Fleischauer, B.W. Shore and K. Bergmann, Adv. At. Mol. Opt. Phys. 46, 55 (2001).

[8] P. Marte, P. Zoller and J.L. Hall, Phys. Rev. A 44, R4118 (1991).

[9] R.G. Unanyan, M. Fleischhauer, B.W. Shore, and K. Bergmann, Opt. Commun. 155, 144 (1998).

[10] H. Theuer, R.G. Unanyan, C. Habscheid, K. Klein, and K. Bergmann, Opt. Express 4, 77 (1999).

[11] R.G. Unanyan, B.W. Shore, and K. Bergmann, Phys. Rev. A 59, 2910 (1999).

[12] B.Y. Chang, I.R. Solá, V.S. Malinovsky, and J. Santamaría, Phys. Rev. A 64, 033420 (2001).

[13] R.G. Unanyan, B.W. Shore, and K. Bergmann, Phys. Rev. A 63, 043401 (2001).
[14] Z. Kis and F. Renzoni, Phys. Rev. A 65, 032318 (2002).

[15] Z. Kis and S. Stenholm, Phys. Rev. A 64, 065401 (2001).

[16] Z. Kis and S. Stenholm, special issue of J. Mod. Optics 49, 111 (2002).

[17] Z. Kis and S. Stenholm, Phys. Rev. A 64, 063406 (2001).

[18] B.W. Shore, J. Martin, M.P. Fewell, and K. Bergmann, Phys. Rev. A 52, 566 (1995).

[19] J. Martin, B.W. Shore, and K. Bergmann, Phys. Rev. A 52, 583 (1995).

[20] J. Martin, B.W. Shore, and K. Bergmann, Phys. Rev. A 54, 1556 (1996).

[21] C.E. Carroll and F.T. Hioe, Phys. Rev. Lett. 68, 3523 (1992).

[22] T. Nakajima, M. Elk, J. Zhang, and P. Lambropoulos, Phys. Rev. A 50, R913 (1994).

[23] N.V. Vitanov and S. Stenholm, Phys. Rev. A 60, 3820 (1999).

[24] L.P. Yatsenko, R.G. Unanyan, K. Bergmann, T. Halfmann, and B.W. Shore, Opt. Commun. 135, 406 (1997).

[25] N.V. Vitanov and S. Stenholm, Phys. Rev. A 56, 741 (1997).

[26] E. Paspalakis, M. Protopapas, and P.L. Knight, Opt. Commun. 142, 34 (1997).

[27] R.G. Unanyan, N.V. Vitanov, and S. Stenholm, Phys. 


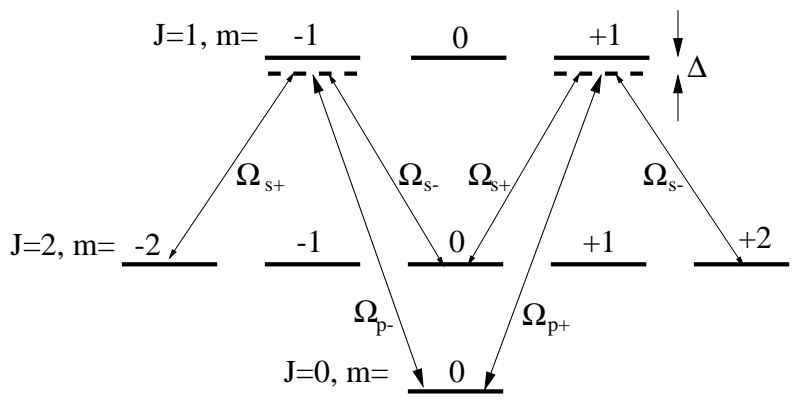

FIG. 1: The six-level $\Lambda$ system. The levels $|2, \pm 1\rangle$ and $|1,0\rangle$ are not coupled to other levels. The remaining levels form the six-level $\Lambda$ configuration. Levels with $J=0$ and $J=2$ are coupled through the levels with $J=1$ by the pump (p) and Stokes (S) laser pulses with right- and left-hand circular polarization $(+/-)$. The corresponding Rabi frequencies are $\Omega_{S \pm}$ and $\Omega_{p \pm}$, respectively. The pulses are at two-photon resonance, but may be detuned from the $J=1$ levels by a certain detuning $\Delta$. Only the level $|0,0\rangle$ is populated initially.

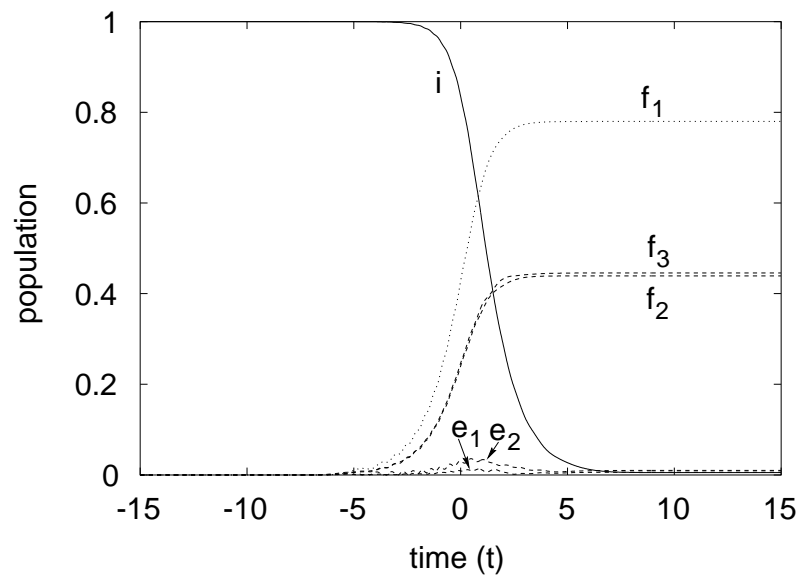

FIG. 2: The numerically computed time evolution of the populations are shown for a set of Gaussian pulses. The pulse shapes are the following: $R_{p}=10 \exp \left[-((t-1.6) / 2.8)^{2}\right]$, $R_{S}=10 \sqrt{1+q^{2}} \exp \left[-((t+1.6) / 2.8)^{2}\right]$; the polarizations are characterized by the angles $\eta=0.5, \nu=1.04$; finally $\varphi=3.34$. The excitation is resonant $\Delta=0$. Time is measured in arbitrary units.
Rev. A 57, 462 (1998).

[28] R.G. Unanyan, N.V. Vitanov, B.W. Shore, and K. Bergmann, Phys. Rev. A 61, 043408 (2000).

[29] B.W. Shore, The theory of coherent atomic excitation (Wiley, New York, 1990).

[30] E. Arimondo, in Progress in Optics ed. E. Wolf, XXXV, p. 257 (Elsevier, Amsterdam, 1996).

[31] Maple is a symbolic computer algebra software, a product of Waterloo Maple Inc. 


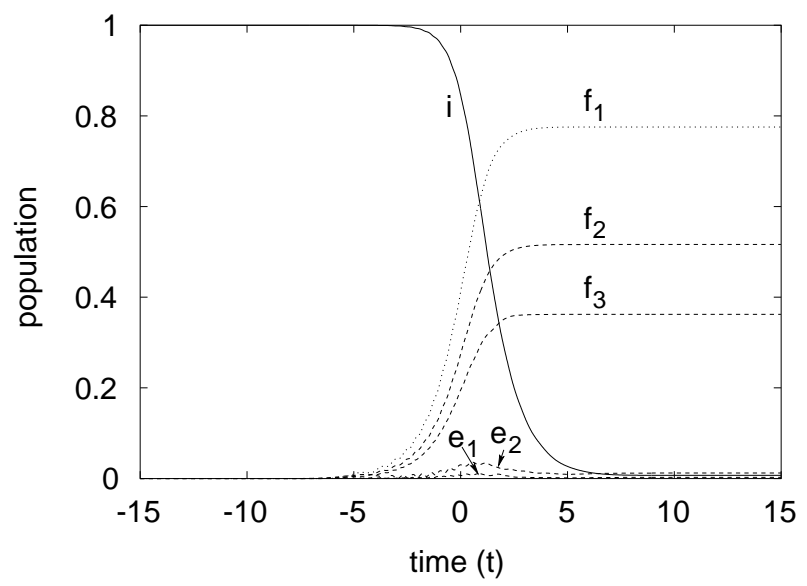

FIG. 3: The numerically computed time evolution of the populations are shown for another set of Gaussian pulses. The pulse shapes are the same as in Fig. 2 only the phase $\varphi$ is changed to 4.74 .
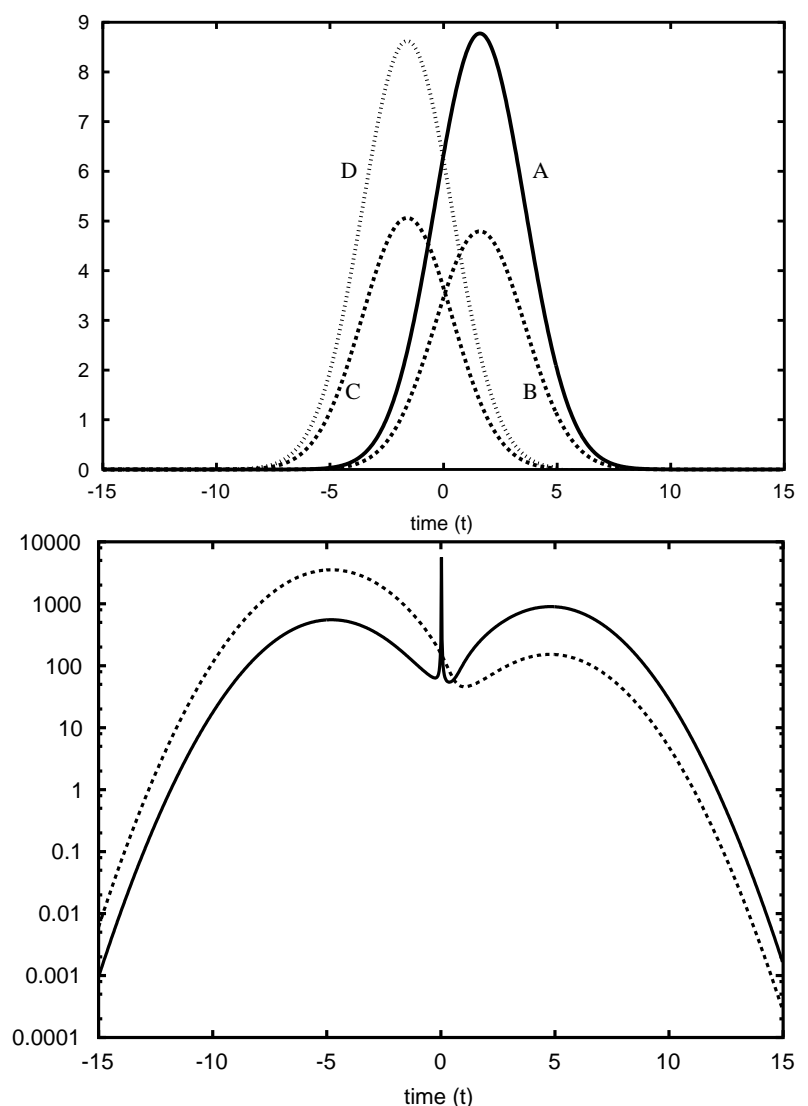

FIG. 4: The adiabaticity of the process shown in Fig. 2] The upper plot shows the pulses, in the lower figure the ratios $\left|\varepsilon_{k}-\varepsilon_{0}\right| /\left|\left\langle D_{2} \mid \dot{B}_{k}\right\rangle\right|, k=1 \ldots 4$ are plotted. Note that two pairs of the eigenstates and matrix elements coincide, hence only two curves can be distinguished. Time and frequency are measured in arbitrary units. 


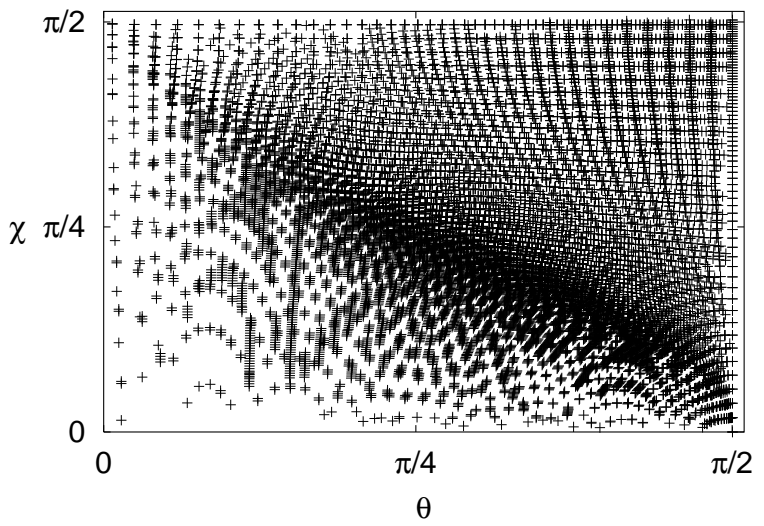

FIG. 5: The slice with $\delta=0.30 \pm 0.05$ of the cube filled by the points $(\theta, \chi, \delta)$ which result from the numerical solution of the Scrödinger equation (6). We have fixed the shape of the pulses and the time delay between them, only their polarizations and relative phases were varied.

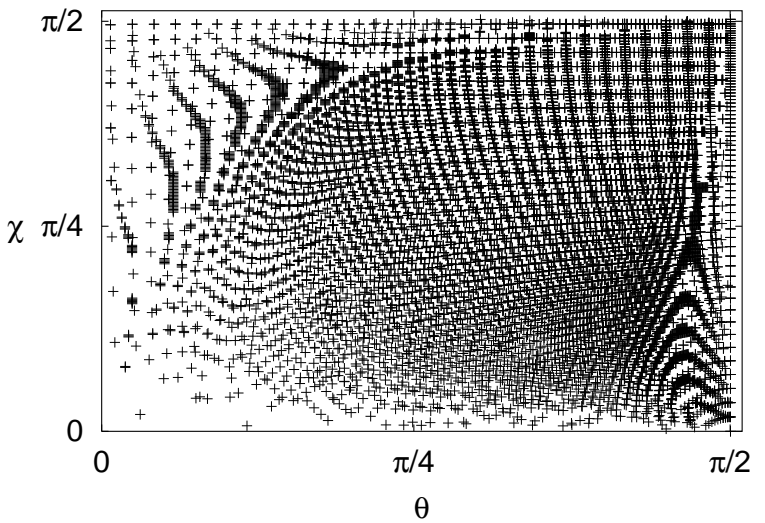

FIG. 6: Same as Fig. 5 but $\delta=2.4$.

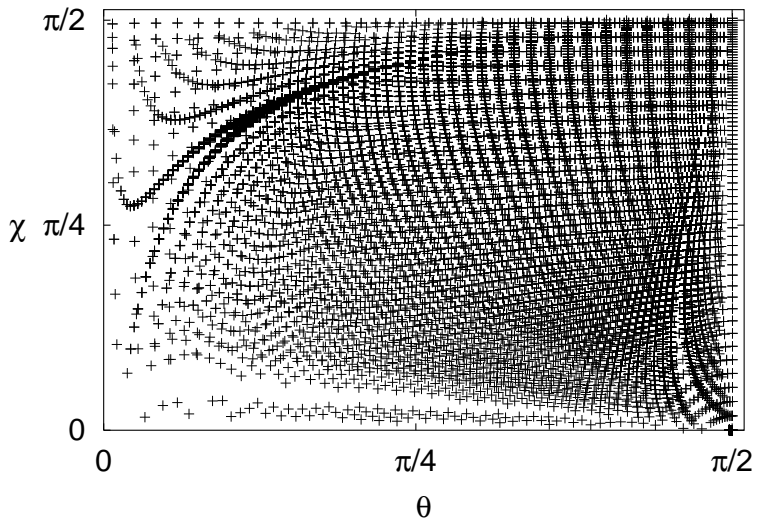

FIG. 7: Same as Fig. 5 but $\delta=3.2$. 


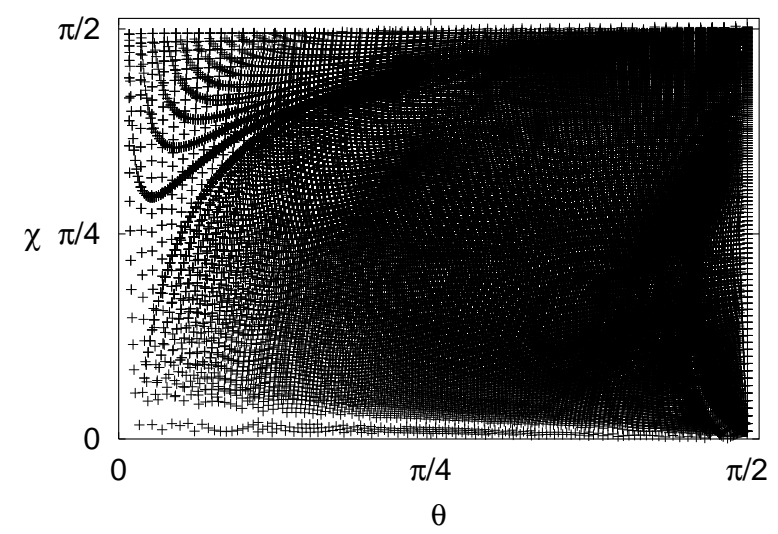

FIG. 8: Same as Fig. 7 but the points are computed from the analytical solution Eq. (24).

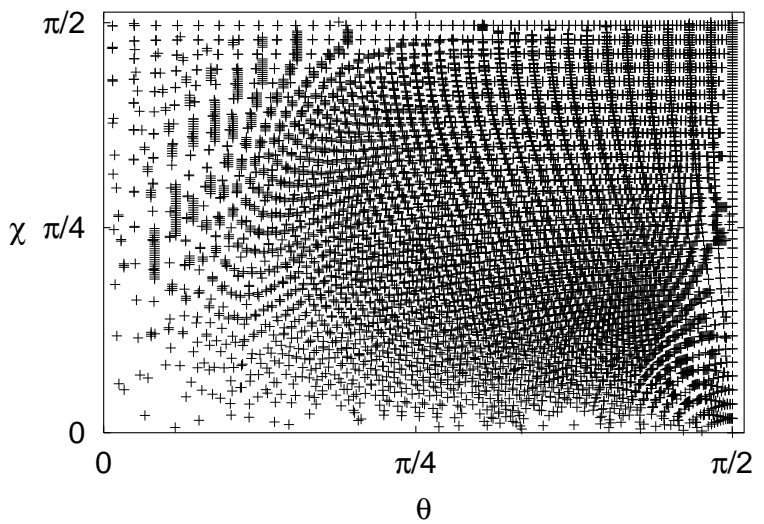

FIG. 9: Same as Fig. 5 but $\delta=4.5$.

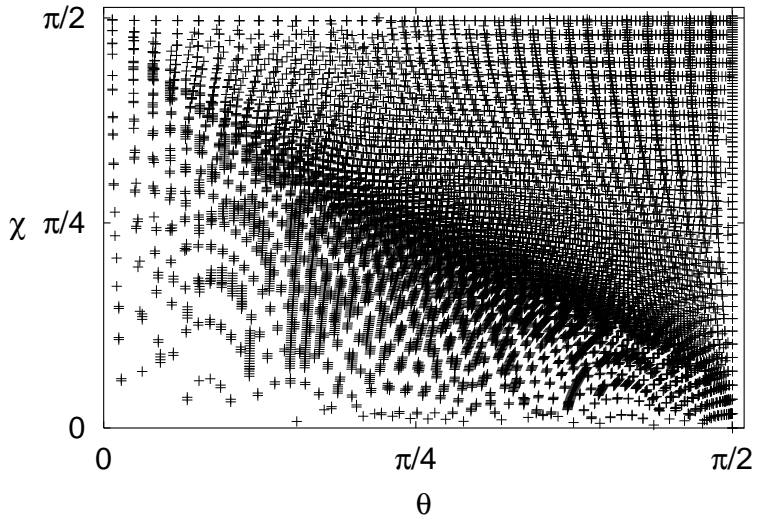

FIG. 10: Same as Fig. 5 but $\delta=6.0$. 